\title{
Prolonged Disease Control with Nivolumab in Metastatic Lung Adenocarcinoma During Immunotherapy Era
}

\author{
Murat Sari and Pinar Saip
}

\begin{abstract}
Immune checkpoint inhibitors have taken an important place in the field of oncologic immunotherapy, especially for patients in whom conventional treatment regimens have failed. Nivolumab prevents programmed cell death 1 (PD-1) and programmed cell death ligand-1 (PD-L1) binding, resulting in blockage of the inhibitory signal and thus facilitating an immunologic antitumor response. Nivolumab has been shown to be effective in thoracic malignancies, including non-small cell lung cancer (NSCLC). Here is a case of 52-year male patient with metastatic lung adenocarcinoma progressing after 4 cycles of chemotherapy. We started nivolumab q2 weeks therapy. Firstly, his dyspnea relieved, carcinoembryonic antigen (CEA) and C-reactive protein (CRP) levels regressed, and then their levels remained constant at a stable level. Radiologically stable response was seen. Progression was seen after about one and half year. Patient died in a short time after progression. In conclusion, this observation provides a strong indication of the potential value of immune checkpoint inhibitors for the management of metastatic lung cancers.
\end{abstract}

Key Words: Nivolumab, Non-small cell lung cancer, Stable response.

\section{INTRODUCTION}

Currently, the options are limited for the treatment of patients who have failed two lines of chemotherapy for advanced non-small cell lung cancer (NSCLC). Nivolumab, causing $T$ cell activation and $T$ cell infiltration to tumor tissue through the blockade of the interaction between programmed cell death 1 (PD-1) and programmed cell death ligand 1 (PD-L1), has been recently clinically applied to NSCLC treatment. ${ }^{1-3}$

\section{CASE REPORT}

A 52-year male, who had no comorbidity, was admitted with a 6-month back pain and enlarged right supraclavicular lymph node in June 2015. He had 30-year history of smoking and no alcohol drinking. A biopsy of the supraclavicular lymph node revealed metastatic lung adenocarcinoma. An epidermal growth factor receptor (EGFR) mutation was positive for de novo T790M, and anaplastic lymphoma kinase (ALK) rearrangement was negative. A full-body positron emission tomographycomputed tomography (PET-CT) revealed a hypermetabolic right lung hilar mass and hypermetabolic right supraclavicular and mediastinal lymphadenopathy, right pleural effusion and osteolytic bone lesion at the T11 vertebral body. His carcinoembryonic antigen (CEA) level was $2314 \mathrm{ng} / \mathrm{ml}$, C-reactive protein (CRP), $185 \mathrm{mg} / \mathrm{dl}$

Department of Medical Oncology, Istanbul University Institute of Oncology, Istanbul, Turkey

Correspondence: Dr. Murat Sari, Department of Medical Oncology, Istanbul University Institute of Oncology, Istanbul, Turkey

E-mail:drmuratsari@gmail.com

Received: March 28, 2018; Accepted: July 06, 2018
$(0-5 \mathrm{mg} / \mathrm{dl})$ and serum albumin $2.8 \mathrm{~g} / \mathrm{dl}$. He was diagnosed with a stage IV disease and received chemotherapy with pemetrexed and cisplatin. Stereotactic body radiotherapy was applied to T11 lytic bone lesion. A computed tomography (CT) scan of the neck and chest after 4 cycles of chemotherapy revealed a stable disease (SD). But CEA level was $3135 \mathrm{ng} / \mathrm{ml}$, and CRP $160 \mathrm{mg} / \mathrm{dl}$. We decided to administer nivolumab (3 $\mathrm{mg} / \mathrm{kg})$ every 2 weeks as a second-line treatment with nivolumab early access programme in November 2015. After the first cycle, his back pain, dyspnea, and fatigue recovered. CRP and CEA levels were regressed to $69 \mathrm{mg} / \mathrm{dl}$ and $635 \mathrm{ng} / \mathrm{ml}$, respectively. After the 6th cycle, CEA level was $68 \mathrm{ng} / \mathrm{ml}$ and there was significant regression in the size of primary as well as metastatic nodes on CT. Interestingly, despite taking nivolumab every two weeks, patient's CRP and CEA levels had always been around $80 \mathrm{mg} / \mathrm{dl}$ and $70 \mathrm{ng} / \mathrm{ml}$, respectively. Also there were always stable responses in images taken three months apart. So, the same treatment was continued with stable response. Seventeen months after the start of nivolumab, the patient's back pain, dyspnea and fatigue developed again and CEA and CRP levels progressed to $110 \mathrm{ng} / \mathrm{ml}$ and $120 \mathrm{mg} / \mathrm{dl}$, respectively. Recurrent pleural effusion, mass and $25 \%$ progression in metastatic lymphadenopathies and new metastatic lesions were seen in thoracic CT. After the patient had been in treatment for 17 months, nivolumab was terminated due to progression of the disease. In the following period, the patient's clinical status deteriorated very quickly and he died in a short time.

\section{DISCUSSION}

Adenocarcinoma of the lung represents approximately $25 \%$ of NSCLC. The first-line treatment for metastatic 
lung adenocarcinoma is platinum-based doublet chemotherapy when driver mutations are negative in our country. When progression occurs, currently, singleagent docetaxel chemotherapy is the standard secondline treatment, resulting in median overall survival (OS) of approximately 7 months. 4 The prognosis for patients who progress after treatment with two or more chemotherapy regimens is very poor. No standard treatments exist for such patients. Nivolumab monotherapy provided clinically meaningful activity with an acceptable side effect profile in our patient and also significantly prolonged survival. Therefore, the discovery of novel drugs is an important task. Immune checkpoint inhibitors may provide tumor regression by reversing tumorinduced immuno-suppression and restoring antitumor

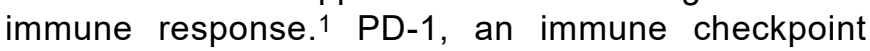
receptor, that negatively regulates T-cell activation, is up-regulated in tumor-infiltrating lymphocytes. Nivolumab is an anti PD-1 monoclonal antibody that inhibits the binding of PD-1 to PD-L1 and PD-L2, thereby attenuating inhibitory signals and enhancing the host antitumour activity. ${ }^{2}$ In a randomized phase III trial, patients received either nivolumab $(3 \mathrm{mg} / \mathrm{kg}$ every 2 weeks, intravenously) or docetaxel $\left(75 \mathrm{mg} / \mathrm{m}^{2}\right.$ every 3 weeks, intravenously). Median OS was 9.2 months in 135 patients in the nivolumab group and 6.0 months in 137 patients in the docetaxel group. ${ }^{3}$ In 2015 , the FDA approved the use of nivolumab in lung adenocarcinoma patients as the second-line therapy for advancedstage. 5 Since then, immunotherapy has been quickly incorporated into our oncology practice and is being considered for incorporation in treatment options for many other cancers. Nivolumab was effective and well tolerated and provided a good long-term stable partial response in our patient. Right now, immunotherapy is being considered for first-line therapy for metastatic disease like lung cancers. In contrast to cytotoxic chemotherapy, hematological toxic effects are infrequently reported with nivolumab. Side effects of nivolmab are mostly tolerable like low-grade treatmentrelated fatigue, decreased appetite, nausea, asthenia, and rash. But despite the above clinical advantages, immunotherapy like nivolumab is much more expensive than conventional chemotherapy. So, many patients may not have access to such successful and less toxic treatments.

\section{REFERENCES}

1. Borghaei H, Lynch TJ, Rizvi NA. A phase III comparative study of nivolumab (anti-PD-1; BMS-963558; ONO-4538) versus docetaxel in patients (pts) with previously treated advanced/ metastatic nonsquamous non-small-cell lung cancer (NSCLC). J Clin Oncol 2013; 31(Suppl):TPS8121.

2. Domingues $D$, Turner $A$, Silva MD. Immunotherapy and lung cancer: current developments and novel targeted therapies. Immunotherapy 2014; 6:1221-35.

3. Rizvi NA, Mazieres J, Planchard D. Activity and safety of nivolumab, an anti-PD-1 immune checkpoint inhibitor, for patients with advanced, refractory squamous non-small-cell lung cancer (CheckMate 063): a phase 2, single-arm trial. Lancet Oncol 2015; 16:257-65.

4. Brahmer J, Reckamp KL, Baas P. Nivolumab versus docetaxel in advanced squamous non-small cell lung cancer. $N$ Engl $J$ Med 2015; 373:123-35.

5. Kazandjian D, Khozin S, Blumenthal G. Benefit-risk summary of nivolumab for patients with metastatic squamous cell lung cancer after platinum-based chemotherapy a report from the US food and drug administration. JAMA Oncol 2016; 2:118-22. 